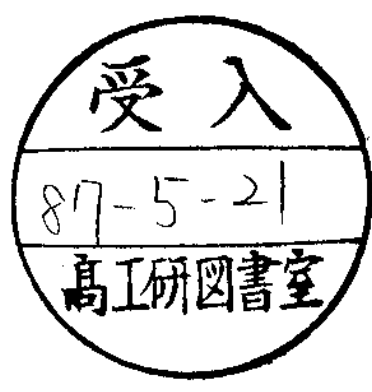

CERN-TH. $4680 / 87$

THERMAL EVENT HORIZONS, MODULI AND BOSON ORERATOR FORMALISM

\title{
ON RIEMANN SURFACES
}

M. Martellini

Dept. di Fisica, Università di Milano

20133 Milano

and

INFN-Sezione di Pavia

and

N. Sánchez ${ }^{*}$ )

CERN - Geneva

\section{A B S T R A C T}

The quantization of a free massless scalar field on an arbitrary compact Riemann surface, as we 11 as the corresponding operator formalism, is developed in terms of analytic mappings with arbitrary critical points. Guided by a multievent horizon Lorentzian picture of the surface, we find the explicit structure of the Fock space states and their dependence on the surface topology. The main feature is that they manifest a "multi-temperature" distribution due to the complicated topological effects. As a by-product, we discuss also the link with the infinite Grassmannian of the Sato theory for the $K-P$ hierarchy and also show how the temperature-like parameters play here the rôle of local moduli for the surface.

*) Permanent address: Observatoire de Paris-Meudon, Groupe d'Astrophysique Relativiste ERl76 CNRS, 92195 Meudon Principal Cedex, France. 
The recent works on the Polyakov functional quantization scheme of string mode $1 \mathrm{~s}^{1)}$ have renewed interest in two-dimensional conformal field theories. As a result, Polyakov's partition function and h-loop amplitudes are given by sums over random surfaces with h-handles. At the critical dimensions, these sums reduce to integrations over the moduli space $M_{h}$ of genus $h$-Riemann surfaces $\Sigma$. From another point of view, string theory is described in perturbation theory in terms of a two-dimensional field theory on $\Sigma$, the fields are the co-ordinates of the string, which in the string tree approximation (i.e., genus $h=0$ ) is a conformally invariant field theory on the twice-punctured sphere described by a virasoro algebra with central extension ${ }^{2)}$. The extension of this approach to higher genus surfaces is far from being clear, essentially because one must combine the main features of the operator formalism, which are "local" concepts, with "global" properties associated to the topology of an arbitrary genus Riemann surface.

In Ref. 3), the virasoro and current algebras are discussed on general Riemann surfaces in the path integral approach, so that the Fock space realization of them still remains quite obscure. Recently, there has been some progress ${ }^{4}$ in the construction of an operator formalism for a free field theory, generically not conformally invariant, on an arbitrary surface by using infinite Grassmannians of the Sato theory ${ }^{5}$ for the Kadomtsev-Petviashvilly (K-P) hierarchy. The basic idea there is that moduli spaces of various genera are included in Sato's universal Grassmannian manifold (UGM) $\operatorname{Gr}(\infty)$ and that the states of the field operators are sections of determinant line bundles on $\operatorname{Gr}(\infty)$.

The interesting approaches of Refs. 4) are unfortunately rather formal in the sense that the Fock space $\mathcal{F}$ as well as the infinite-rank Kac-Moody group $G L(\infty)$ of canonical transformations acting on $\mathcal{F}$ depend on the topology of the surface only implicitly. In other words, one does not know the explicit structure of the states of $\mathcal{F}^{\prime}$ and how they depend on the genus of the Riemann surface. On the other hand, it is reasonable to think that the effects of the topology are similar to those produced by a "thermal reservoir", i.e., the non-trivial topological background maintains a certain number of excited states which manifest themselves as "holes" in the ground state" . If this is actually the case, the structure of the Fock space $\mathcal{F}$ should be very rich and complicated.

The results in this letter show that $\mathcal{F}^{\prime}$ is spanned by "particle" and "quasiparticle" states whose spectrum depends on the topology of the surface. Furthermore, notice that it will be possible to discuss the usual operator calculations, 
Feynman diagram techniques and renormalization questions (in the operator formalism) only if the explicit structure of the sectors of $\mathcal{F}_{\text {is }}$ known.

The general framework advocated in this letter is essentially that of quantum field theory in terms of analytic (holomorphic) mappings with critical points developed by one of the authors ${ }^{7)}$ in the context of the Hawking-Unruh mechanism ${ }^{8}$ ). The key idea here is that the explicit construction of the Fock space $\widetilde{F}$ of a two-dimensional free massless field theory on an arbitrary surface of genus $h>0$ is obtained by quantizing on a multiply-connected two-dimensional space-time endowed with $q=2 h$-event horizons. Roughly speaking, this is a "Lorentzian" representation of the "Euclidean" quantization of a free field theory on a compact closed Riemann surface $\Sigma$ of genus $h$.

In this letter we provide also the connection with Sato's UGM approach ${ }^{5)}$ and show that the temperature-1ike parameters labelling the "conformal-vacua" over $\Sigma$ play the rôle of modular parameters ${ }^{9}$ ) for the surface $\Sigma$. In the following we shall indicate the basic points of our approach, which we shall apply to a free massless scalar field.

A) Conformal mappings in the formal Euclidean continuation of the Minkowski space-time $[i . e .,(x, t) \rightarrow(x, i \tau)]$ are represented by analytic functions

$$
\begin{array}{ll}
\mu \rightarrow \mu^{\prime}=F(\mu) & \\
\mu^{\prime} \rightarrow \mu=f\left(\mu^{\prime}\right) \quad\left(f \equiv F^{-1}\right)
\end{array}
$$

$(u \equiv x+i \tau, \bar{u} \equiv x-i \tau)$ : We require these mappings to satisfy

$$
f( \pm \infty)=\mu \pm
$$

where $u_{ \pm}$can take finite or infinite values. In Minkowski space, the mapping $f$ represents a transformation from an inertial frame $(x, t)$ to an accelerated one $\left(x^{\prime}, t^{\prime}\right)$. Constant values of $x^{\prime}$ define the world-lines of the accelerated observers in the $(x, t)$ plane. Real points where $f^{\prime}\left(u^{\prime}\right)=0$ are critical points of the transformation and determine event horizons in the Minkowski space-time. These are the boundaries $u=u_{ \pm}$of the space-time domain over which $t h e\left(x^{\prime}, t^{\prime}\right)$ manifold is defined. This is so because the two families of characteristic lines $x \pm t=$ const. $=c$ coalesce upon going to Euclidean space to the real points $(c, 0)$; in particulax, the frontiers of the light cone coalesce to the origin. 
B) As is known, two triangulated compact surfaces are topologically equivalent if and only if they agree in character of orientability, number of contours and Euler characteristic $\chi=2-2 h-b$ with $h(b)$ being the number of handles (boundaries). An important consequence of this is that we can construct simple model surfaces corresponding to the canonical forms. Consider, for instance, a circular disk in which we make h circular holes. The double of this region is a closed orientable surface and, in various simple ways, we can construct an explicit triangulation whose characteristic turns out to be $2-2 \mathrm{~h}$. Hence it represents a surface with $b=0$ and the given $h$. It is visualized as a sphere with $h$ handles, which is the topological type of a closed orientable Riemann surface of genus $h$. Then our underlying idea is that a two-dimensional free quantum field theory on a closed $(b=0)$ surface with $g$-handles may be equivalently regarded as defined on a circular disk $(h=0)$ with $b=2 g$-holes cut out. Notice that in string theory, model surfaces with the same Euler characteristic describe the same dual diagram, since the string's loop expansion parameter is just the Euler characteristic. Once stated this picture, one may describe in Minkowski space the above Euclidean quantum field theory in terms of "accelerated observers" defined by mappings $f\left(u^{\prime}\right)$ having an arbitrary number of real critical points. Indeed, in the Euclidean representation, the set of points $M=D-\left\{p_{1}, \ldots, p_{2}\right\}$ where $D$ is the domain, say a disk, of the conformal boost $u^{\prime}=F(u)$ and $p_{1}, \ldots, p_{2 h}$ are $q \equiv 2 h$ real critical points of $u=f\left(u^{\prime}\right)$ (see below), is homotopically a surface $\Sigma$ with $q$ circular holes around the points $P_{i}$ which describes, as we have observed, a closed surface of genus $h=q / 2$. Mappings $f\left(u^{\prime}\right)$ having two real critical points (i.e., $h=1$ ) one at $x=u_{-}$and another at $x=u_{+}>u_{-}$define accelerated observers in the rhombus $u_{-} \leqslant|x \pm t| \leqslant u_{+}$of Minkowski space-time. In this case, there are two event horizons. Furthermore, a mapping $f\left(u^{\prime}\right)$ with $2 h-c r i t i c a l$ points may be represented by a multi-valued function such that each branch $f^{i}\left(u^{\prime}\right)$ $(i=1,2, \ldots, h)$ has exactly two real critical points. The different accelerated manifolds cover causally disjointed regions

$$
u_{-}^{(i)} \leqslant|x \pm t| \leqslant \mu_{+}^{(i)}
$$

of Minkowski space-time. In our approach, the branching degree $i$ plays the same rôle as the genus $h$ in the Euclidean picture. 
c). The "toy model" of a conformal map which describes a manifold having 2h-event horizons is given by the h-branches of the inverse function of the trans formation

$$
\mu^{\prime}=F(\mu)=\log \frac{\left(\mu-\mu_{-}\right)^{p}}{\left(\mu_{+}-\mu^{q}\right.} \quad, \mu_{-}^{(h)} \leqslant \mu \leqslant \mu_{+}^{(h)}
$$

Here $p$ and $q$ are positive constants which, as we will see, characterize the quantum spectrum of the theory. In particular, the well-known Rindler space with one event horizon (at $u=0$ ) is defined by the mapping

$$
\mu^{\prime}=F(\mu)=\frac{1}{\alpha} \log \mu, 0,5 \mu \leqslant \infty(5)
$$

Near $u_{-}$and $u_{+}, F(u)$ [Eq. (4)] defines the imaginary time $\tau^{\prime}$ as being periodic with period $\beta_{-}=2 \pi \mathrm{p}$ and $\beta_{+}=2 \pi q$ respectively; similarly for $\tau^{\prime}$ defined by Eq. (5) with period $\beta=2 \pi / \alpha$. The quantum propagators of the free fields in the $\left(x^{\prime}, \tau^{\prime}\right)$ manifold exhibit the same periodicity in the imaginary time $\tau^{\prime}$, indicating the presence of thermal features at temperatures $\left(\beta_{-}\right)^{-1}$ and $\beta_{+}^{-1}$ respectively. Each branch $f^{i}\left(u^{\prime}\right)$ of $f$ entails two characteristic parameters $T_{ \pm}^{(i)}$ $(i=1, \ldots, h)$

$$
T_{ \pm}^{(i)}=\frac{1}{2 \pi} \frac{d}{d u^{\prime}}\left[\log f^{i}\left(\mu^{\prime}\right)\right] \mu^{\prime}= \pm \infty,
$$

which in the Fock representation of the theory play the rôle of temperatures. The differential winding number of the mapping at the critical points gives the temperatures of the vacuum spectrum ${ }^{7}$ ).

D) The Fock representation of the two-dimensional free massless scalar quantum fields in the accelerated manifolds is obtained entirely in terms of the mappings ${ }^{7}$. Bogoliubov transformations relate the positive frequency $(\lambda)$ modes with respect to the accelerated time $t$ ' to the positive frequency (k) modes with respect to the inertial time $t$. The Bogoliubov coefficients $A_{\lambda k}$ and $B_{\lambda k}$ are expressed as (modified) Laplace transformations with the kernel determined by $F(u)$ of the accelerated wave functions, e.g., 


$$
\begin{aligned}
& B_{\lambda k}=\frac{1}{2 \pi}\left(\frac{k}{\lambda}\right)^{1 / 2} \mathcal{L}_{\lambda}(-i k), A_{\lambda k}=i B_{\lambda_{0}-k} \\
& \mathcal{L}_{\lambda}(k)=\int_{u_{-}}^{u+} d u e^{-k \mu-i \lambda F(\mu)}
\end{aligned}
$$

A characteristic vacuum spectrum is given by the quantity

$$
\begin{aligned}
N\left(\lambda, \lambda^{\prime}\right) & =\sum_{k} B_{\lambda}(k) B_{\lambda^{\prime}}^{*}(k)= \\
& =\frac{1}{4 \pi^{2}} \frac{1}{\sqrt{\lambda \lambda^{\prime}}} \iint_{\mu_{-}}^{\mu_{+}} \frac{d u d u^{\prime}}{\left(\mu-\mu^{\prime}+i \varepsilon\right)^{2}} e^{\left[i \lambda F(u+i \varepsilon)-i \lambda^{\prime} F\left(\mu^{\prime}-i \varepsilon\right)\right]}
\end{aligned}
$$

which describes the number of the $\lambda$ modes in the inertial Minkowski vacuum. This quantity is invariant under the bilinear transformations $F(u)=(\alpha u+\beta / \gamma u+\delta)$ with $(\alpha \delta-\beta \gamma) \neq 0$. [All the accelerated frames defined in an orbit of the $0(2,1)$ group have the same vacuum spectra.] Knowledge of $F(u)$ determines entirely the properties of the conformal or thermal vacuum $\left|0^{\prime}\right\rangle \equiv \mid 0\left(T_{ \pm}^{(i)}-1\right\rangle$. Conversely, given $N\left(\lambda, \lambda^{\prime}\right)$ one can reconstruct the mapping. The different $N\left(\lambda, \lambda^{\prime}\right)$ and their associated temperatures can be classified according to the different types of singularities of $F(u)$.

D.i) We show now how the temperature-1ike parameters $\beta_{ \pm}^{(i)} \equiv\left(T_{+}^{(i)}\right)^{-1}$ have a nice geometrical interpretation in terms of local modular parameters ${ }^{g}$ ) for the compact Riemann surface $\Sigma$ of genus $i$ "modelled" on the conformal boost domain $M=D-\left\{p_{1}, \ldots, P_{2}\right\}$ previously discussed. Furthermore, we shall show how our thermal vacuum $\left|O\left(\beta_{ \pm}^{(i)}\right)\right\rangle$ associated to $\sum$ corresponds to a point of Sato's UGM, $G r(\infty) 5)$. For this purpose, let us consider only the simpler case of a single temperature-like parameter $\beta=T^{-1}$ and review the construction of $\left.10(\beta)\right\rangle$ of Ref. 7) with slight modifications in notations.

In the two-dimensional Minkowski space-time, massless scalar field operators are defined by

$$
\psi=\sum_{n} \psi_{n} e^{-i n \mu}, \psi^{*}=\sum_{n} \psi_{n}^{*} e^{i n v}
$$

in terms of null variables $u=x-t, v=x+t$. [we may map the $(u, v)-s$ pace onto the complex $p l$ ane by $a$ Wick rotation $t \rightarrow i \tau$ and then by $z=\exp (\tau+i x)$, so that 
$\exp (-i u n) \rightarrow z^{-n}$ and $\exp (i v n) \rightarrow \bar{z}^{-n} l$. The canonical commutation relations (CCR) are

$$
\left[\psi_{n}, \psi_{m}^{*}\right]=\delta_{n m}, \quad\left[\psi_{n}, \psi_{m}\right]=\left[\psi_{n}^{*}, \psi_{m}\right]=0
$$

and the Fock (no-particle) vacuum state is given by $\psi_{-n}|0\rangle=\psi_{n}^{*}|0\rangle=0(n>0)$. As usual, the field operators, Eqs. (10), span a Fock space $\mathcal{F}$ out of the vacuum 10). Let us now consider the analogous quantization in the non-inertial frame described by the null co-ordinates $u^{\prime} \equiv x^{\prime}-t^{\prime}, v^{\prime} \equiv x^{\prime}+t^{\prime}$ with $x \pm t=f\left(x^{\prime} \pm t^{\prime}\right)(f$ as defined above). The field operators $\psi, \psi^{*}$ in terms of the positive frequencies with respect to the non-inertial time $t^{\prime}$ read

$$
\psi=\sum_{m} \psi_{m}^{\prime} e^{-i m \mu^{\prime}}, \psi^{*}=\sum_{m} \psi_{m}^{\prime *} e^{i m v^{\prime}}
$$

with the CCR given by

$$
\left.\left[\psi_{\Omega}^{\prime}, \psi_{s}^{*}\right]=\delta_{r s} \quad ; \quad \psi_{\Omega}^{\prime} \psi_{s}^{\prime}\right]=\left[\psi_{\Omega}^{*}, \psi_{s}^{\prime *}\right]=0
$$

The operators $\psi_{\mathrm{m}}^{\prime}, \psi_{\mathrm{m}}^{\prime *}$ define a so-called conformal ("thermal") vacuum state as $\psi_{-\mathrm{m}}^{\prime}|0\rangle=\phi_{\mathrm{m}}^{\prime *}\left|0^{\prime}\right\rangle=0(\mathrm{~m}>0)$, but which is not really a ground state (since it is excited with respect to $|0\rangle)$. We observe here that the Bogoliubov transformation of the *- algebra, Eq. $\left.(10)^{*}\right)$ generates a GL $(\infty)$-automorphism of the above algebra defined by

$$
\begin{aligned}
\psi_{m}^{\prime} & =\cosh \left[\theta_{m}(\beta)\right] \psi_{m}+\sinh \left[\theta_{m}(\beta)\right] \psi_{-m} \\
& =\exp (-i \hat{G}(\beta)) \psi_{m} \exp (i \hat{G}(\beta)), \forall_{m} \in \mathbb{z}^{(13)}
\end{aligned}
$$

and analogously for $\psi_{m}^{\prime *}$. [Here the Bogoliubov coefficient $\theta_{m}(\beta)$ is a function of the free parameter $m \in Z$ and $\beta \in \mathbb{R}$. l The generator $G(\beta)$ of this transformation is found to be

$$
\hat{G(\beta)}=-\frac{i}{2} \sum_{n} \theta_{n}(\beta)\left[\psi_{n} \psi_{-n}^{*}-\psi_{-n} \psi_{n}^{*}\right]
$$

Since the Bogoliubov transformation [Eq. (13)] leaves the total Hamiltonian invariant, the generator $\widehat{G}(\beta)$ is conserved. One can now take advantage of the

*) The Bogoliubov transformations generate *- automorphisms [GL( $\infty)$ ] in the so-called Weyl algebra defined by Eq. (12) and play the same rôle as the *automorphisms in the Clifford algebra for the fermion system of Ref. 10). 
freedom of this transformation and define equivalently the Fock space $F$ by the conformal-vacuum state $\left|0^{\prime}\right\rangle$ which can also be written in the canonical form

$$
\left|0^{\prime}\right\rangle \equiv|O(\beta)\rangle=\exp (-i \hat{G}(\beta))|0\rangle
$$

In this operator formalism, the Bogoliubov transformations $g \equiv \exp (-i G(\beta))$

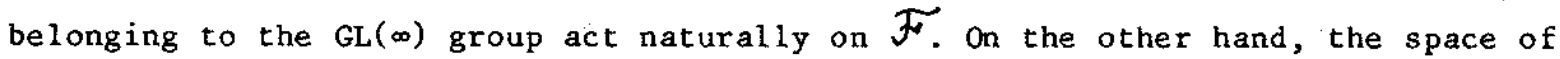

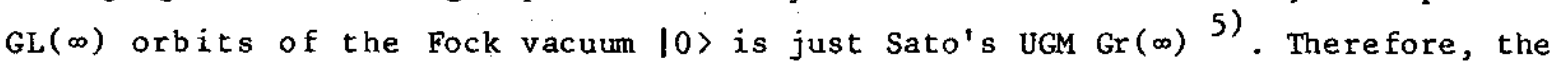
conforma1 vacuum $|O(\beta)\rangle=g|0\rangle$, Eq. (15), for each $g \in G L(\infty)$ corresponds to a point of $\mathrm{Gr}(\infty)$. By this infinite Grassmannian $\operatorname{Gr}(\infty)$, one may also clarify the relation between our approach and the more abstract formalism of Ref. 10).

As a consequence of these results, the correlation functions of the surface $\Sigma \sim \mathrm{D}-\left\{\mathrm{P}_{1}\right\}$ [a Euclidean representation of the so-called Rindler space, Eq. (5)] are given, following the Schwinger-DeWitt formalism ${ }^{11}$ ), by taking matrix elements of free-boson operators between the "in-vacuum" $<$ in, $01 \equiv<01$ and the "out-vacuum" $\mid 0$, out $\rangle \equiv|0(\beta)\rangle$. In particular, the vacuum-to-vacuun persistence amplitude $\langle$ in, 0$| 0$, out $\rangle \equiv\langle 0 \mid O(\beta)\rangle^{*}$ ) gives the so-called boson partition function $z(\beta)$ [in this case $\beta \equiv \mathrm{T}^{-1}=\alpha / 2 \pi$ where $\alpha$ is defined in Eq. (5)]. On the other hand, in a Polyakov-like approach ${ }^{12}$ ) restricted to this case, $\mathrm{Z}(\beta)$ is given formally by a path-integral over free massless scalar fields defined on the annulus $\Sigma \sim D-\left\{P_{1}\right\}$ and $\beta$ is naturally associated with a local (real) modular parameter describing inequivalent shapes of the annulus $\Sigma$. Thus, we argue that in our operator formalism the temperature-1ike parameters $\beta_{ \pm}^{(i)}$ labelling different Bogoliubov transformations $\exp \left(-i G\left(\beta_{ \pm}^{(i)}\right)\right)$ have in the associated path integral approach a geometrical interpretation as "moduli parameters".

E) In the operatorial formalism presented in the previous sections, we see that the Fock space of a two-dimensional free massless scalar quantum field theory for the cases I) genus $h=0$, II) genus $h=1$, III) genus $h>2$ are essentially different since they correspond to the following classification.

*) For a fermion system, $\langle 0 \mid 0(\beta)\rangle$ is known as the so-called $\tau$ function at zero "infinite" times for a point $|O(\beta)\rangle$ in $\operatorname{Gr}(\infty)$. 


\begin{tabular}{|c|c|c|}
\hline GENUS & MAPPING & FOCK SPACE (BASIC FEATURE) \\
\hline I) $h=0$ & $\begin{array}{l}F(u) \text { has power and/or } \\
\text { essential singularities } \\
\text { at } u=u_{ \pm}= \pm \infty \text {. }\end{array}$ & $\begin{array}{l}\text { Non-thermal spectrum } \\
\text { ("particle states") } \\
{ }_{ \pm}=0 \\
{ }^{\mathrm{B}_{\lambda k} \mathrm{k} \dot{+}_{\infty}} \mathrm{e}^{-\mathrm{k}} \rightarrow 0 \\
\left\langle\mathrm{~T}_{\mu \nu}\right\rangle{ }_{\mathrm{u}} \mathrm{u}_{ \pm} 0\end{array}$ \\
\hline II) $h=1$ & $\begin{array}{l}F(u) \text { has logarithmic sin- } \\
\text { gularities at } u=u_{-}<\infty \\
\text { and at } u=u_{+}<\infty[\text { say } \\
F(u) \text { as in Eq. (4)] }\end{array}$ & $\begin{array}{l}\text { There exists a double- } \\
\text { thermal spectrum ("quasi- } \\
\text { particle states") } \\
\mathrm{N}\left(\lambda, \lambda^{\prime}\right)=\left(\delta \lambda \lambda^{\prime} / 2\right)\left[1 / \mathrm{e}^{2 \pi \lambda \mathrm{p}_{-1}+}\right. \\
+1 / \mathrm{e}^{\left.2 \pi \lambda \mathrm{q}_{-1}\right]} \\
\mathrm{T}_{-}=1 / 2 \pi \mathrm{p}, \mathrm{T}_{+}=1 / 2 \pi \mathrm{q} \\
\mathrm{B}_{\lambda \mathrm{k} \mathrm{k} \rightarrow \infty} \mathrm{k}^{i \lambda / \sqrt{\mathrm{k}}} \\
\left\langle\mathrm{T}_{\mu \nu}\right\rangle \underset{\mathrm{u} \rightarrow \mathrm{u}_{ \pm}}{ }(\pi / 6) \mathrm{T}_{ \pm}^{2}\end{array}$ \\
\hline III) $h>2$ & $\begin{array}{l}\text { As in case (II) but now } \\
\text { we consider h-branches } \\
\text { of the inverse function } \\
\text { of } \mathrm{Eq} \text {. ( } 4 \text { ). }\end{array}$ & $\begin{array}{l}\text { Multi-thermal spectrum. We } \\
\text { have different inequivalent } \\
\text { "strange" representations } \\
\text { the CCR. }\end{array}$ \\
\hline $\begin{array}{l}{\left[\text { Here }\left\langle\mathrm{T}_{\mu \nu}\right\rangle\right.} \\
\text { momentum }\end{array}$ & tands for the expectatio & $\begin{array}{l}\text { alue of the energy- } \\
\text {.] }\end{array}$ \\
\hline
\end{tabular}

F) Let us consider the case III. Here we have, roughly speaking, h-inequivalent (uncorrelated) Fock spaces $\mathcal{F}_{ \pm}^{(h)}$ : each pair $\mathscr{F}_{ \pm}^{(h)}$ describes a degenerate "strange" representation of the CCR (in which the vacuum state contains an 
infinite number of particles) and it is essentially a "copy" of the Fock spaces $\mathscr{H}_{ \pm}$associated to the case II $(h=1)$. Such a situation reminds us of the physical interpretation of Haag's theorem ${ }^{13)}:$ if the equal-time CCR makes sense at all, then inequivalent strange representations of the CCR must be realized at different "times" t defined by $u_{-}(h) \leqslant|x \pm t|_{x=c o n s t} \leqslant u_{+}^{(h)}$. Then, one may set $\tilde{F}_{\text {tot }}=\oplus\left(\mathcal{F}_{+}^{(h)} \oplus \mathcal{F}_{-}^{(h)}\right)$. An explicit realization of a manifold with 2 h-event horizons (i.e., 2h-holes) is provided by the general mapping.

$$
\prod_{i=1}^{h} \frac{\left(\mu_{-\mu_{-}}\right)^{p_{i}}}{\left(\mu_{+}^{i}-\mu^{q_{i}}\right.} P_{(\mu)}=\cdots f\left(\mu^{i}\right)
$$

such that

$$
f^{\prime}( \pm \infty)=0
$$

The inverse mapping $u^{\prime}=F(u)$ has $2 h$ singularities (at $u=u_{-}^{i}$ and $u^{\prime}=u_{+}^{i}$, $i=1,2, \ldots, h)$ of strengths $\mathrm{p}_{i}$ and $\mathrm{q}_{\mathbf{i}}$ respectively; $\mathrm{p}_{\mathbf{i}}$ and $\mathrm{q}_{\mathbf{i}}$ are positive constants and $P(u)$ is an analytic function without real zeros and poles. In particular, it $c$ an be $P(u)=$ constant. The mappings

$$
\frac{\left(\mu_{-}-\mu_{-}\right)^{p}}{\left(\mu_{+}-\mu^{q}\right.}=f\left(\mu^{\prime}\right)
$$

(with two event horizons) and

$$
u=f\left(\mu^{\prime}\right)
$$

(with one event horizon) are particular cases of Eq. (16) for $h=1$ and particular values of $p$ and $q$. The mapping $f\left(u^{\prime}\right)=e^{\alpha u^{\prime}} y i e l d s$.

$$
u^{\prime}=\log \prod_{i=1}^{h} \frac{\left(\mu-\mu_{-}^{i}\right)^{p_{i}}}{\left(\mu_{+}^{i}-\mu^{q_{i}}\right.} P(\mu)
$$

i.e.,

$$
\left\{\begin{array}{l}
\mu^{\prime}=\sum_{i=1}^{h} F^{i}(\mu)+\log P_{(\mu)} \\
F^{i}=\log \frac{(\mu-\mu-i) p_{i}}{\left(\mu_{t}^{i}-\mu\right) q_{i}}
\end{array}\right.
$$

In Minkowski space, we have h causally disconnected regions ( $h$ rhombus) and thus have h-double Fock representations [h copies, one pair $\mathcal{F}_{ \pm}^{(i)}$ for each rhombus 
(i)]. For each rhombus (i) we have a set of Bogoliubov coefficients $A_{\lambda k}^{(i)}, B_{\lambda k}^{(i)}$ and a set of temperatures $T_{-}^{(i)}=1 / 2 \pi \mathrm{P}_{i}, \mathrm{~T}_{+}^{(i)}=1 / 2 \pi \mathrm{q}_{i}$ characterizing the vacuum spectrum $N^{(i)}\left(\lambda, \lambda^{\prime}\right)$ (and the same features as already discussed in our classification follow). We should require identification of the h-rhombus, and thus describe the quantum mechanics of a multiply-connected space ${ }^{14)}$. After "identifications", the Fock representations of the spaces with $h=l$ and with $h>l$ are essentially different. It can be pointed out that our h-rhombuses are real worlds and not merely $h$ images (or $h$ "mirror-universes") of one real world; $h$ "mirror-worlds" will be defined by the mapping

$$
\prod_{i=1}^{h} \frac{\left(\mu_{-1} \mu_{-}^{i}\right)^{p_{i}}}{\left(\mu_{+}^{i}-\mu^{q_{i}}\right.} P(\mu)=-f\left(\mu^{\prime}\right),
$$

which provides the analytic (complete) extension for the negative $x$ 's. Even if the identification of our h-rhombus leads to analogies with the "elliptic picture" of space-time" 15 ), note that in the "elliptic interpretation", one identifies the real world with its mirror (time-reversed) images. In this sense, the problems of the elliptic picture in quantum mechanics, namely the presence of zero-norm states, do not appear in our picture. Our Fock representation obtained after identification is perfectly well defined.

We also note the following differences between the case $h=1$ (two horizons) and the case $h>1$ (multi-horizons): even if for each rhombus (i) ( $i=1, \ldots, h)$ we have essentially the same thermal features as in the case of one rhombus $(h=1)$, note that $B_{\lambda k}^{i} \neq B_{\lambda k}^{l} \forall(\lambda, k), N^{i}\left(\lambda, \lambda^{\prime}\right) \neq N^{l}\left(\lambda, \lambda^{\prime}\right) \forall\left(\lambda, \lambda^{\prime}\right)$ and $\left\langle T_{\mu \nu}^{i}\right\rangle \neq\left\langle T_{\mu \nu}^{l}\right\rangle \forall(u, v)$.

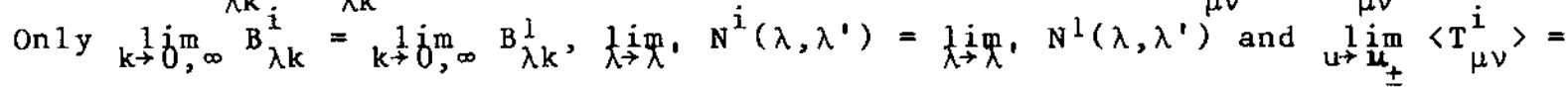
$\lim _{u \rightarrow u_{ \pm}}\left\langle T_{\mu \nu}^{1}\right\rangle$. Note that global thermal equilibrium (i.e. $\left.\left\langle T_{\mu \nu}\right\rangle=(\pi / 6) T^{2}+k, v\right)$ is only possible in the presence of only one event horizon. Going from one to two event horizons breaks thermal equilibrium (even if $\mathrm{T}_{+}=\mathrm{T}_{-}$). In the case with $2 \mathrm{~h}$ event horizons there is a local (i.e., asymptotic) thermal equilibrium situation (in other words, $\partial / \partial t^{\prime}$ is not a killing vector globally, but only asymptotically for $u \rightarrow u_{ \pm}$.

At the end, it should be noted that ultra-violet divergences arise in the free energy (F) and entropy (S) of quantum fields on a given manifold, from the existence of an event horizon in the space-time. (The same problem appears in the case of a four-dimensional black hole.) $S$ and $F$ need the presence of an ultra-violet cut-off $\Lambda$. This regularization reflects the fact that a classical fixed geometry is no longer valid at distances of order of the planck length lp, 
so that $\Lambda \sim \log (1 / \ell \mathrm{p})$. This is equivalent to considering a shift of the horizon with a length cut-off $\varepsilon \sim$ lp in the mapping

$$
\mu^{\prime}=F(\mu+\varepsilon)
$$

covering a domain $u_{-}^{(h)}\left\langle|x \pm t+\varepsilon|<u_{+}^{(h)}\right.$.

In the context of strings, our manifolds $(x, t)$ and $\left(x^{\prime}, t^{\prime}\right)$ can be regarded, as we have seen, as representing different (inequivalent) dual graphs, respectively, a string-tree diagram and a string-loop diagram at the order $h=q / 2$, where $q$ is the (even) number of critical points of $f\left(x^{\prime} \pm t^{\prime}\right)$. Then one can show that the presence of the cut-off $\varepsilon$ is crucial in order to get a finite string length in $\left(x^{\prime}, t^{\prime}\right)$ and as a consequence, a discrete mass spectrum. Further details will be presented elsewhere ${ }^{16)}$.

\section{ACKNOWLEDGEMENTS}

We thank L. Alvarez-Gaume, H.J. de Vega and C. Reina for discussions. 


\section{REFERENCES}

1) For reviews and detailed references, see, e.g.:

L. Alvarez-Gaune and P. Nelson - CERN Preprint TH. 4615 (1986).

2) A.A. Belavin, A.M. Polyakov and A.B. Zamolodchikov - Nucl.Phys. B241 (1984) 331 ;

D. Friedan, E. Martinec and S. Shenker - Nucl.Phys. B271 (1986) 93;

E. Martinec - Nucl.Phys. B281 (1987) 157.

3) T. Eguchi and H. Ooguri - Nucl.Phys. B282 (1987) 308;

H. Sonoda - Nuc1. Phys. B281 (1987) 546.

4) L. Alvarez-Gaume, C. Gomez and C. Reina - CERN Preprint TH. 4641 (1987);

A.A. Beilinson, Yu.I. Manin and Y.A. Shechtman - in preparation;

N. Ishibashi, Y. Matsuo and H. Ooguri - Univ. of Tokyo Preprint UT-499 (1986);

S. Saito - Tokyo Metropolitan Univ. Preprint TMUP-HEL-8613 (1986).

5) M. Sato and Y. Sato - in Non-Linear Partial Differential Equations in Applied Science, Eds. H. Fujita, P.D. Lax and G. Strang (North Holland, Amste rdam, 1983);

G. Segal and G. Wilson - Publications of the IHES, 61 (1985).

6) V.G. Kac and D.H. Peterson - Publications of the IHES, 63 (1985).

7) N. Sánchez - Phys.Rev. D24 (1981) 2100; Phys.Lett. 105B (1981) 375; Phys.lett. 81A (1981) 424; in Proc. 2nd Marcel Grossmann Meeting, Ed. R. Ruffini (North Holland, Amsterdam, 1982), Vo1. 2, p. 501.

8) See, for example: N.D. Birrell and P.C.W. Davies - "Quantum Field Theory in Curved Space-Time", CUP, Cambridge, U.K. (1982) and references therein.

9) See, for example: W. Abikoff - "The Real Analytic Theory of Teichmuller Space", Springer Verlag (1980).

10) N. Ishibashi, Y. Matsuo and H. Ooguri - in Ref. 4);

S. Saito - in Ref. 4).

11) See, for example: B.S. DeWitt - Physics Reports 19C (1975) 297.

12) D. Friedan and S. Shenker - Nuc1.Phys. B281 (1987) 509.

13) See, for example: A.S. Wightman - in High Energy Electromagnetic Interactions and Field Theory, Cargèse Lectures (1964), Vol. 2, Ed. M. Levy, Gordon and Breach (1967).

14) J.S. Dowker - J.Phys. A: Gen.Phys. 5 (1972) 936.

15) G.W. Gibbons - Nuc1.Phys. B271 (1986) 497;

N. Sánchez and B.F. Whiting - Nucl.Phys. B283 (1987) 605.

16) H. de Vega and N. Sánchez - in preparation. 